\title{
Hypertoniebehandlung
}

\section{Neue invasive Therapien}

Die letzten Jahre haben viel Bewegung in die Hypertoniebehandlung gebracht. Neben der bewährten medikamentösen Therapie sind eine Reihe von alternativen Verfahren entwickelt worden, wie zum Beispiel die Impfung, aber auch invasive Verfahren, die der vorliegende Hypertonie-Schwerpunkt zum Thema hat. Die neuen Verfahren sind zunächst für die resistente Hypertonie entwickelt worden, haben aber alle auch das Potenzial für eine breitere Anwendung, falls die positiven Erfahrungen über einen längeren Zeitraum hinweg reproduzierbar sind und negative Spätfolgen aus„Die neuen invasiven Therapien haben das Potential zur breiten Anwendung positive Langzeitergebnisse vorausgesetzt." hung der Hypertonie sowohl epidemiologisch als auch pathophysiologisch gut belegt ist, kann die nCPAP-Therapie doch in vielen Fällen den bestehenden Bluthochdruck nicht in größerem Ausmaß senken. Die Gründe hierfür sind nur teilweise bekannt.

Dervorliegende Hypertonie-Schwerpunkt soll mit den neuen Verfahren bekannt machen, nicht aber die unkritische Anwendung propagieren. Speziell beider renalen Denervation ist bereits jetzt zu befürchten, dass ähnlich wie bei anderen invasiven Prozeduren die Machbarkeit über die Anwendung entscheiden könnte. Die Pround Kontra-Diskussion in diesem Schwerpunkt mag hilfreich sein, zu einer rationellen Anwendung dieses Verfahrens zu gelangen.

Ihr

Walter Zidek 